\title{
Conservative Treatment versus Percutaneus Wire Fixation in Treatment of Distal Radial Fracture in Elderly
}

\author{
Mohammed Al-Morsi, Mohamed Ebrahim, Abdelfatah El-Senosy
}

Department of Orthopedic, Benha faculty of medicine, Benha University, Egypt.

Correspondence to: Abdelfatah El-Senosy, Department of Orthopedic, Benha faculty of medicine, Benha University, Egypt.

Email:

drelsenosy@yahoo.com

Received: 5 January 2020

Accepted: 28 March 2020

\begin{abstract}
Distal radius fractures are commonly encountered in orthopedic practice especially in elderly patients. A number of clinical papers have supported the idea that anatomic restoration of the distal end of the radius is essential to gain superior results. The aim of the study is to systematically review the literature for management of distal end radius in elderly persons (conservative treatment versus percutaneous wire fixation) as regards radiological and functional outcomes. Studies were identified from the Medline, Cochrane, EMBASE, and Google Scholar databases were searched until, 2019 using combinations of the following search terms: distal radius fracture, conservative treatment, non-operative treatment, and nonsurgical treatment, surgical treatment, operative, elderly, and older. Reference lists of relevant studies were manually searched. There was no statistical significance difference between CI and PKF groups' frequency of complication in all of the selected studies. Based on
\end{abstract} results we recommend for more analysis regarding every parameter of the radiographic and functional results and specific complication related to each fixation need to be accomplished, which requires more RCTs with high quality. Surgical treatment seems to be more effective distal radius fracture compared with conservative treatment when the radiographic outcomes were analyzed, and no significant differences were detected in the functional outcomes and complication rate.

Keywords: radius, fracture, Surgical

CI: Cast immobilization PKF: Percutaneous K wire fixation 


\section{Introduction}

Distal end radius fracture is a common fracture type and has an approximate incidence of 1:10 000 people and represents $17 \%$ of all skeletal fractures. ${ }^{(1)}$ Distal radius fracture is one of the most frequent fracture human injuries in elderly patients, which represents about $17 \%$ of all the skeletal fractures. ${ }^{(2)}$

Distal radius fractures represent one of the most common injuries treated by upper extremity surgeons, accounting for $16 \%$ of all fractures treated in the emergency room in the United States and $75 \%$ of fractures of the forearm. The age distribution for injuries to the distal radius is typically bimodal with peaks in the 5-14 year age group and in elderly patients older than 60 . Most distal radius fractures occur in elderly females with a male-to-female ratio of 1:4. ${ }^{(3,4)}$

Multiple newer classifications have improved Orthopedists' understanding of fracture patterns and injury severity. Descriptive classifications, such as the Malone and Frykman classifications, provide detailed anatomic fracture pattern analysis. The well-established AO classification with its subgroups may help improve documentation. The injury mechanism-based fracture classification according to Fernandez and Jupiter assigns fractures to bending, shearing, or compressive force mechanisms. (4) The importance of anatomic reduction has been demonstrated by various clinical studies as well as laboratory assessment of the force and stress loading across the radiocarpal joint. ${ }^{(1)}$

Distal radius fractures in osteoporotic bone have greatly diminished stability; there is often bone impaction and fracture fragmentation. Surgical fixation is more difficult because the fixation device cannot gain as robust and reliable hold as in younger, good quality bone. As one would expect, fractures in the elderly therefore tend to displace. ${ }^{(\mathbf{5 , 6})}$

Although ample literature supports good short-term outcomes, higher-level evidence examining the outcomes of surgical fixation in distal radial fractures stresses the importance of age and socioeconomic status. ${ }^{(7)}$

No evidence supports one surgical technique over the other in younger patients, and no consensus exists regarding the best treatment for these fractures in elderly patients. ${ }^{(\mathbf{8})}$ The choice of the treatment depends on many factors such the patients' age, life style, type of the facture, severity and alignment of the fracture, condition of the soft tissues. ${ }^{(2)}$ 


\section{Materials and methods}

Literature search strategy: Studies were identified from the Medline, Cochrane, EMBASE, and Google Scholar databases were searched from 2011 to 2019 using combinations of the following search terms: distal radius fracture, conservative treatment, non-operative treatment, and nonsurgical treatment, surgical treatment, operative, elderly, and older. Reference lists of relevant studies were manually searched.

Selection criteria: Studies were selected for inclusion in the analysis based on the following criteria: Reported or compared treatment outcomes between nonsurgical and surgical treatment of distal radius fractures. Randomized controlled trial (RCT), comparative prospective, or retrospective study. Reported quantitative clinical and radiological outcomes. Letters, comments, editorials, case reports, and non-English publications were excluded. Articles that did not specify the type of conservative or surgical treatment were also excluded. All included reviews were approved by ethical committee of Benha faculty of medicine.

Data extraction: The following information/data were extracted from studies that met the inclusion criteria: name of the first author, year of publication, study design, number of participants in each treatment group, participants' age and gender, type of intervention for each group, Disabilities of the Arm, Shoulder and Hand (DASH) and visual analogue scale (VAS) pain scores for study and control groups, functional assessment by grip strength and ranges of wrist motion.

\section{Outcome measures and data analysis:}

The primary outcome measure was DASH scores of surgical and nonsurgical groups, which is based on a questionnaire of symptoms and disability, and best reflects the subjective functional outcomes felt by the patients. Secondary outcomes were VAS pain scores and objective functional assessments (including grip strength and active range of wrist motion) and radiographic parameters (including graduating inclination and ulnar variance) of the two groups. The standard difference in post-treatment means was calculated for the outcomes of the surgical and nonsurgical treatment groups.

Statistical Analysis: For this systematic review, it was considered that the number of fractures, the rate of intra-articular fractures, means patient age and the length of the follow-up time, as potential sources of heterogeneity and analyzed these factors using analysis of variance (ANOVA) and the Chi square test. 
The weighted means of continuous outcome measures were calculated across available studies for each treatment option. Wrist motion, grip strength, radiographic parameters, and DASH scores from available articles were pooled together by generating the weighted means for all factors. Significance level was set at $p=0.05$. If significant differences were detected, multiple comparisons of both treatment options were performed using Multiple Comparisons test.

\section{Results}

The studies were collected from different countries (Egypt, China, USA, London, Taiwan and India). All studies include cases with extra-articular fracture. The intervention in all studied was CI or PKF. The range number of cases was from thirty to more than four hundreds in all ten studies. Seven studies include relatively small number of cases (less than one hundred), while three of them involve more than one hundred up to approximately five hundred cases ).Table (1).

This table shows that there was no statistical significance difference between $\mathrm{CI}$ and PKF groups in mean age or sex distribution in any of the selected studies. Table (2)

This table shows that there was no statistical significance difference between $\mathrm{CI}$ and PKF groups in extension, flexion or pronation in any of the selected studies. Table (3)

This table shows that there was statistical significant difference between $\mathrm{CI}$ and PKF groups in radial inclination, ulnar variance and volar tilt in most of the selected studies who reported their parameters. In view of radial inclination difference, two studies found high significant difference, while five studies found significant difference. In view of ulnar variance difference, four studies found significant difference, one study found highly significant difference, while one study found no significant difference and four studies did not report any difference. In view of volar tilt difference, two studies found high significant difference, while two studies found significant difference; however, no difference was reported by the remaining six studies. Three studies did not report any difference in the whole three radiological parameters. Table (4)

This table shows that there was no statistical significance difference between $\mathrm{CI}$ and PKF groups in grip strength in all the selected studies, except one study. Table (5)

This table shows that there was no statistical significant difference between $\mathrm{CI}$ and PKF groups in different evaluation systems in six studies. However, the four remaining studies showed high significant 
difference in between the two treatment options Different scoring systems were used as functional outcome score which was used by five studies, pain score system was used by one study, visual analogue score by one study, Gartland and Werley score was used by one study, and the remaining two studies used the daily activity as a scoring system. Table (6).

Table (1): Type of intervention among the selected studies.

\begin{tabular}{|c|c|c|c|c|c|}
\hline Authors (year) & Country & Race & Type of fracture & Intervention & $\begin{array}{l}\text { No of } \\
\text { cases }\end{array}$ \\
\hline Refai et al,( 2019) & Egypt & Egyptian & $\begin{array}{l}\text { Unstable extra } \\
\text { articular }\end{array}$ & $\begin{array}{l}-\mathrm{CI} \\
-\mathrm{PKF}\end{array}$ & 30 \\
\hline $\begin{array}{l}\text { El-Adawy et al } \\
(2018)\end{array}$ & Egypt & Egyptian & Extra articular & $\begin{array}{l}-\mathrm{CI} \\
-\mathrm{PKF}\end{array}$ & 70 \\
\hline $\begin{array}{l}\text { Konde et al } \\
\text { (2018) }\end{array}$ & India & Indian & $\begin{array}{l}\text { Unstable extra } \\
\text { articular }\end{array}$ & $\begin{array}{l}-\mathrm{CI} \\
-\mathrm{PKF}\end{array}$ & 60 \\
\hline Vasudevan, et al ( 2018) & USA & American & $\begin{array}{l}\text { Unstable extra } \\
\text { articular }\end{array}$ & $\begin{array}{l}-\mathrm{CI} \\
-\mathrm{PKF}\end{array}$ & 496 \\
\hline Rastogi, et al (2018) & India & Indian & $\begin{array}{l}\text { Unstable extra } \\
\text { articular }\end{array}$ & $\begin{array}{l}\text { CI } \\
\text {-PKF }\end{array}$ & 40 \\
\hline $\begin{array}{l}\text { Khan,et al } \\
\text { ( 2017) }\end{array}$ & Taiwan & Taiwanes & $\begin{array}{l}\text { Unstable extra } \\
\text { articular }\end{array}$ & $\begin{array}{l}-\mathrm{CI} \\
\text {-PKF }\end{array}$ & 30 \\
\hline oVenkatesh et al (2016). & India & Indian & Extra articular & $\begin{array}{l}-\mathrm{CI} \\
-\mathrm{PKF}\end{array}$ & 60 \\
\hline Ju et al.,2015 & China & Chines & $\begin{array}{l}\text { Unstable extra } \\
\text { articular }\end{array}$ & $\begin{array}{l}-\mathrm{CI} \\
\text {-PKF }\end{array}$ & 237 \\
\hline Bajwa et al.,(2015). & $\begin{array}{l}\text { London and } \\
\text { Cambridge }\end{array}$ & England & $\begin{array}{l}\text { Unstable extra } \\
\text { articular }\end{array}$ & $\begin{array}{l}\text { CI } \\
\text {-PKF }\end{array}$ & 50 \\
\hline Diaz-Garcia et al.,(2011) & USA & American & Extra articular & $\begin{array}{l}-\mathrm{CI} \\
-\mathrm{PKF}\end{array}$ & 401 \\
\hline
\end{tabular}

Table (2): Demographic data among the selected studies.

\begin{tabular}{|c|c|c|c|c|c|c|}
\hline \multirow[b]{2}{*}{ Authors (year) } & \multicolumn{2}{|c|}{ Age } & \multicolumn{4}{|c|}{ Gender } \\
\hline & $\begin{array}{c}\text { CI } \\
\text { Mean } \pm \text { SD } \\
\end{array}$ & $\begin{array}{c}\text { PKF } \\
\text { Mean } \pm \text { SD }\end{array}$ & P1 & $\begin{array}{c}\mathbf{C I} \\
\mathbf{M} / \mathbf{F} \\
\end{array}$ & $\begin{array}{l}\text { PKF } \\
\mathbf{M} / \mathbf{F} \\
\end{array}$ & $\mathbf{P 2}$ \\
\hline Refai et al,(2019) & $61 \pm 10.1$ & $62 \pm 9.2$ & $\begin{array}{l}>0.05 \\
\mathrm{NS}\end{array}$ & $9 / 6$ & $8 / 7$ & $\begin{array}{l}>0.05 \\
\mathrm{NS}\end{array}$ \\
\hline El-Adawy et al (2018) & $61 \pm 4$ & $60 \pm 5$ & $\begin{array}{l}>0.05 \\
\mathrm{NS}\end{array}$ & $21 / 14$ & $19 / 16$ & $\begin{array}{l}>0.05 \\
\mathrm{NS}\end{array}$ \\
\hline Konde et al (2018) & $65 \pm 7$ & $63 \pm 6.2$ & $\begin{array}{l}>0.05 \\
\mathrm{NS}\end{array}$ & $9 / 21$ & $7 / 23$ & $\begin{array}{l}>0.05 \\
\mathrm{NS}\end{array}$ \\
\hline Vasudevan, et al (2018) & $64 \pm 6$ & $66 \pm 8$ & $\begin{array}{l}>0.05 \\
\mathrm{NS}\end{array}$ & $140 / 111$ & $138 / 107$ & $\begin{array}{l}>0.05 \\
\mathrm{NS}\end{array}$ \\
\hline Rastogi, et al (2018) & $65.9 \pm 7$ & $69.9 \pm 7$ & $\begin{array}{l}>0.05 \\
\mathrm{NS}\end{array}$ & $8 / 7$ & $11 / 14$ & $\begin{array}{l}>0.05 \\
\mathrm{NS}\end{array}$ \\
\hline Khan,et al ( 2017) & $60.3 \pm 7.1$ & $63.9 \pm 6.3$ & $>0.05 \mathrm{NS}$ & $9 / 6$ & $8 / 7$ & $\begin{array}{l}>0.05 \\
\mathrm{NS}\end{array}$ \\
\hline Venkatesh et al(2016). & $\mathrm{NR}$ & $\mathrm{NR}$ & --- & NR & NR & --- \\
\hline Ju et al.,2015 & NR & $\mathrm{NR}$ & --- & NR & NR & --- \\
\hline Bajwa et al.,(2015). & $60.3 \pm 7.1$ & $63.9 \pm 6.3$ & $>0.05 \mathrm{NS}$ & $16 / 9$ & $11 / 14$ & $\begin{array}{l}>0.05 \\
\mathrm{NS}\end{array}$ \\
\hline Diaz-Garcia et al., (2011) & $65 \pm 6$ & $66 \pm 8$ & $\begin{array}{l}>0.05 \\
N S\end{array}$ & $36 / 203$ & $50 / 112$ & $\begin{array}{l}>0.05 \\
\mathrm{NS}\end{array}$ \\
\hline
\end{tabular}


Table (3): Clinical outcome parameters in the final follow up in the selected studies.

\begin{tabular}{|c|c|c|c|c|c|c|c|c|c|}
\hline Authors (year) & $\begin{array}{l}\text { Extension } \\
\text { CI } \\
\text { Mean } \pm \text { Sd }\end{array}$ & $\begin{array}{l}\text { PKF } \\
\text { Mean } \pm \text { Sd }\end{array}$ & $\mathbf{P}$ & $\begin{array}{l}\text { Flexion } \\
\text { CI } \\
\text { Mean } \pm \text { Sd }\end{array}$ & $\begin{array}{l}\text { PKF } \\
\text { Mean } \pm \text { Sd }\end{array}$ & $\mathbf{P}$ & $\begin{array}{l}\text { Pronation } \\
\text { CI } \\
\text { Mean } \pm \text { Sd }\end{array}$ & $\begin{array}{l}\text { PKF } \\
\text { Mean } \pm \text { Sd }\end{array}$ & $\mathbf{P}$ \\
\hline $\begin{array}{l}\text { Refai et al, } \\
\text { (2019) }\end{array}$ & NR & NR & --- & NR & NR & --- & NR & NR & --- \\
\hline $\begin{array}{l}\text { El-Adawy et al } \\
\text { (2018) }\end{array}$ & $71 \pm 8$ & $72 \pm 7$ & $\begin{array}{l}>0.05 \\
\mathrm{NS}\end{array}$ & $72 \pm 10$ & $73 \pm 8.2$ & $\begin{array}{l}>0.05 \\
\mathrm{NS}\end{array}$ & $75 \pm 7$ & $77 \pm 4$ & $\begin{array}{l}>0.05 \\
\mathrm{NS}\end{array}$ \\
\hline $\begin{array}{l}\text { Konde et al } \\
\text { (2018) }\end{array}$ & $54.6 \pm 14.9$ & $54.8 \pm 18.7$ & $\begin{array}{l}>0.05 \\
\mathrm{NS}\end{array}$ & $51.8 \pm 11.1$ & $47.8 \pm 13.1$ & $\begin{array}{l}>0.05 \\
\mathrm{NS}\end{array}$ & $84.4 \pm 3.8$ & $82.9 \pm 6.8$ & $\begin{array}{l}>0.05 \\
\mathrm{NS}\end{array}$ \\
\hline $\begin{array}{l}\text { Vasudevan, et al } \\
\text { (2018) }\end{array}$ & NR & NR & --- & NR & NR & --- & NR & NR & --- \\
\hline $\begin{array}{l}\text { Rastogi, et al } \\
\text { (2018) }\end{array}$ & $61 \pm 7$ & -- & --- & $57 \pm 10$ & --- & --- & $85 \pm 8$ & --- & --- \\
\hline $\begin{array}{l}\text { Khan,et al } \\
\text { ( 2017) }\end{array}$ & $65 \pm 7.5$ & $61.9 \pm 12.4$ & $\begin{array}{l}>0.05 \\
\mathrm{NS}\end{array}$ & $60.8 \pm 9.5$ & $58.6 \pm 11.5$ & $\begin{array}{l}>0.05 \\
\mathrm{NS}\end{array}$ & $78.2 \pm 7.5$ & $81.3 \pm 7.1$ & $\begin{array}{l}>0.05 \\
\mathrm{NS}\end{array}$ \\
\hline $\begin{array}{l}\text { Venkatesh et al } \\
\text { (2016). }\end{array}$ & 63 & 64.5 & $\begin{array}{l}>0.05 \\
\mathrm{NS}\end{array}$ & 61 & 62 & $\begin{array}{l}>0.05 \\
\mathrm{NS}\end{array}$ & 61 & 64.5 & $\begin{array}{l}>0.05 \\
\mathrm{NS}\end{array}$ \\
\hline Ju et al., 2015 & NR & NR & --- & NR & NR & --- & NR & NR & --- \\
\hline $\begin{array}{l}\text { Bajwa et } \\
\text { al.,(2015). }\end{array}$ & $62 \pm 8.5$ & $60.9 \pm 11.1$ & $\begin{array}{l}>0.05 \\
\mathrm{NS}\end{array}$ & $63.8 \pm 10.5$ & $59.6 \pm 12.5$ & $\begin{array}{l}>0.05 \\
\mathrm{NS}\end{array}$ & $76.2 \pm 8.5$ & $82.1 \pm 8.1$ & $\begin{array}{l}>0.05 \\
\mathrm{NS}\end{array}$ \\
\hline $\begin{array}{l}\text { Diaz-Garcia et } \\
\text { al., (2011) }\end{array}$ & NR & NR & --- & NR & NR & --- & NR & NR & --- \\
\hline
\end{tabular}

Table (4): Clinical outcome parameters in the final follow up in the selected studies.

\begin{tabular}{|c|c|c|c|c|c|c|c|c|c|}
\hline $\begin{array}{l}\text { Authors } \\
\text { (year) }\end{array}$ & $\begin{array}{l}\text { Supination } \\
\text { CI } \\
\text { Mean } \pm \text { Sd }\end{array}$ & $\begin{array}{l}\text { PKF } \\
\text { Mean } \pm \text { Sd }\end{array}$ & $\mathbf{P}$ & $\begin{array}{l}\text { Radial de } \\
\text { CI } \\
\text { Mean } \pm S \\
\text { d }\end{array}$ & $\begin{array}{l}\text { iation } \\
\text { PKF } \\
\text { Mean } \pm S \\
\text { d }\end{array}$ & $\mathbf{P}$ & $\begin{array}{l}\text { Ulnar de } \\
\text { CI } \\
\text { Mean } \pm S \\
\text { d }\end{array}$ & $\begin{array}{l}\text { ation } \\
\text { PKF } \\
\text { Mean } \pm S \\
\text { d }\end{array}$ & $\mathbf{P}$ \\
\hline $\begin{array}{l}\text { Refai et } \\
\text { al,(2019) }\end{array}$ & NR & NR & --- & NR & NR & --- & NR & NR & --- \\
\hline $\begin{array}{l}\text { El-Adawy } \\
\text { et al(2018) }\end{array}$ & $74 \pm 9$ & $75 \pm 6$ & $\begin{array}{l}>0.05 \\
\text { NS }\end{array}$ & $21 \pm 7$ & $20 \pm 6$ & $\begin{array}{l}>0.05 \\
\mathrm{NS}\end{array}$ & $21 \pm 7$ & $20 \pm 6$ & $\begin{array}{l}>0.05 \\
\mathrm{NS}\end{array}$ \\
\hline $\begin{array}{l}\text { Konde et } \\
\text { al (2018) }\end{array}$ & $81.9 \pm 4$ & $82.6 \pm 8.1$ & $\begin{array}{l}<0.03 \\
\mathrm{~S}\end{array}$ & $\begin{array}{l}21.9 \pm 12 \\
4\end{array}$ & $17.2 \pm 7.8$ & $\begin{array}{l}>0.05 \\
\mathrm{NS}\end{array}$ & $31.4 \pm 8.1$ & $28.9 \pm 8.8$ & $\begin{array}{l}>0.05 \\
\text { NS }\end{array}$ \\
\hline $\begin{array}{l}\text { Vasudevan } \\
\text {, et al } \\
(2018)\end{array}$ & NR & NR & --- & NR & NR & --- & NR & NR & --- \\
\hline $\begin{array}{l}\text { Rastogi, et } \\
\text { al (2018) }\end{array}$ & $\begin{array}{l}72.50+6.3 \\
9\end{array}$ & $\begin{array}{l}67.50+5.0 \\
0\end{array}$ & $\begin{array}{l}<0.03 \\
\mathrm{~S}\end{array}$ & $25 \pm 7$ & --- & --- & $35 \pm 8$ & ---- & --- \\
\hline $\begin{array}{l}\text { Khan,et al } \\
\text { (2017) }\end{array}$ & 66.5 & 69.33 & $\begin{array}{l}<0.03 \\
S\end{array}$ & $22.6 \pm 7.5$ & $16.5 \pm 8.1$ & $\begin{array}{l}<0.03 \\
\mathrm{~S}\end{array}$ & $25.7 \pm 8.5$ & $31.2 \pm 7.1$ & $\begin{array}{l}<0.03 \\
S\end{array}$ \\
\hline $\begin{array}{l}\text { Venkatesh } \\
\text { et al } \\
(2016) \text {. }\end{array}$ & 64 & 67 & $\begin{array}{l}>0.05 \\
\mathrm{NS}\end{array}$ & 16 & 17 & $\begin{array}{l}>0.05 \\
\mathrm{NS}\end{array}$ & 22 & 23 & $\begin{array}{l}>0.05 \\
\mathrm{NS}\end{array}$ \\
\hline $\begin{array}{l}\text { Ju et al., } \\
2015\end{array}$ & $78.8 \pm 6.9$ & $80.5 \pm 7.3$ & $\begin{array}{l}>0.05 \\
\mathrm{NS}\end{array}$ & $22.6 \pm 7.5$ & \pm & $\begin{array}{l}>0.05 \\
\mathrm{NS}\end{array}$ & $28.7 \pm 8.5$ & $31.2 \pm 7.1$ & $\begin{array}{l}>0.05 \\
\mathrm{NS}\end{array}$ \\
\hline $\begin{array}{l}\text { Bajwa et } \\
\text { al.,(2015). }\end{array}$ & $79.5 \pm 7.9$ & $80.5 \pm 9.3$ & $\begin{array}{l}>0.05 \\
\mathrm{NS}\end{array}$ & $25.6 \pm 8.5$ & \pm & $\begin{array}{l}>0.05 \\
\mathrm{NS}\end{array}$ & $29.7 \pm 8.5$ & $35.2 \pm 8.1$ & $\begin{array}{l}>0.05 \\
\mathrm{NS}\end{array}$ \\
\hline $\begin{array}{l}\text { Diaz- } \\
\text { Garcia et } \\
\text { al.,(2011) }\end{array}$ & NR & NR & --- & NR & NR & --- & NR & NR & --- \\
\hline
\end{tabular}


Benha medical journal vol. 37, Special issue (surgery), 2020

Table (5): Grip strength in the final follows up in the selected studies.

\begin{tabular}{llll}
\hline Authors (year) & $\begin{array}{l}\text { Grip strength } \\
\text { CI } \\
\text { Mean } \pm \text { SD }\end{array}$ & $\begin{array}{l}\text { PKF } \\
\text { Mean } \pm \text { SD }\end{array}$ & P \\
\hline Refai et al,(2019) & NR & NR & --- \\
El-Adawy et al (2018) & NR & NR & --- \\
Konde et al (2018) & $27.9 \pm 14.3$ & $39 \pm 16.1$ & $<0.01$ \\
Vasudevan, et al ( 2018) & $83 \pm 12.3$ & & HS \\
Rastogi, et al (2018) & & $84 \pm 10$ & $>0.05$ \\
Khan,et al ( 2017) & $18.8 \pm 5.8$ & & NS \\
Venkatesh et al (2016). & $15 \pm 7.1$ & --- & -- \\
Ju et al.,2015 & & $16.13 \pm 6.5$ & $>0.05$ \\
Bajwa et al.,(2015). & NR & NR & NS \\
\hline
\end{tabular}

Table (6): Evaluation of the cases at the final follows up in the selected studies.

\begin{tabular}{|c|c|c|c|c|}
\hline Authors (year) & Score & $\begin{array}{l}\text { Evaluation } \\
\text { CI }\end{array}$ & PKF & $\mathbf{P}$ \\
\hline \multirow[t]{4}{*}{ Refai et al,(2019) } & \multicolumn{4}{|l|}{ Functional outcome score } \\
\hline & Good & $8(53.3)$ & $7(46.6)$ & $>0.05 \mathrm{NS}$ \\
\hline & Fair & $6(40)$ & $7(46.6)$ & $>0.05 \mathrm{NS}$ \\
\hline & Poor & $1(7.7)$ & $1(7.7)$ & $>0.05 \mathrm{NS}$ \\
\hline \multirow{5}{*}{$\begin{array}{l}\text { El-Adawy et al } \\
\text { (2018) }\end{array}$} & \multicolumn{4}{|l|}{ Functional outcome score } \\
\hline & Excellent & 5 & 4 & $<0.01 \mathrm{HS}$ \\
\hline & Good & 12 & 11 & \\
\hline & Fair & 4 & 6 & \\
\hline & Poor & 14 & 14 & \\
\hline \multirow{5}{*}{$\begin{array}{l}\text { Konde et al } \\
(2018)\end{array}$} & \multicolumn{4}{|l|}{ Functional out come score } \\
\hline & Excellent & 7 & 21 & $<0.01 \mathrm{HS}$ \\
\hline & Good & 23 & 9 & \\
\hline & Fair & 0 & 0 & \\
\hline & Poor & 0 & 0 & \\
\hline \multirow{2}{*}{$\begin{array}{l}\text { Vasudevan, et al } \\
\text { (2018) }\end{array}$} & Daily activity unil. & $7.6 \pm 8$ & $7.4 \pm 8$ & $>0.05 \mathrm{NS}$ \\
\hline & Daily activity bil. & $9.7 \pm 12$ & $9.4 \pm 12$ & $>0.05 \mathrm{NS}$ \\
\hline Rastogi, et al (2018) & $\begin{array}{l}\text { Gartland and Werley } \\
\text { demerit point }\end{array}$ & $(13.05+5.14)$ & $(8.35+4.39)$ & $<0.01 \mathrm{HS}$ \\
\hline \multirow{5}{*}{$\begin{array}{l}\text { Khan,et al } \\
\text { ( 2017) }\end{array}$} & \multicolumn{3}{|l|}{ Functional out come score } & $<0.01 \mathrm{HS}$ \\
\hline & Exc ellent & 13 & 8 & \\
\hline & Good & 2 & 7 & \\
\hline & Fair & 0 & 0 & \\
\hline & Poor & 0 & 0 & \\
\hline \multirow{5}{*}{$\begin{array}{l}\text { Venkatesh et al } \\
(2016) .\end{array}$} & \multicolumn{4}{|l|}{ Functional outcome score } \\
\hline & Excellent & 13 & 11 & \\
\hline & Good & 9 & 13 & $>0.05$ \\
\hline & Fair & 7 & 5 & NS \\
\hline & Poor & 1 & 1 & \\
\hline Ju et al.,2015 & Pain score & $0.7 \pm 1.4$ & $0.8 \pm 1.3$ & $>0.05 \mathrm{NS}$ \\
\hline Bajwa et al.,(2015). & $\begin{array}{l}\text { visual analogue score } \\
\text { (VAS) }\end{array}$ & $\begin{array}{l}8.6 \pm 4.2, \\
\text { Range (4-10). }\end{array}$ & $7.6 \pm 3.2$ & $>0.05 \mathrm{NS}$ \\
\hline Diaz-Garcia et al., & Daily activity unil. & $7.6 \pm 8$ & $7.4 \pm 8$ & $>0.05 \mathrm{NS}$ \\
\hline & Daily activity bil. & $9.7 \pm 12$ & $9.4 \pm 12$ & $>0.05 \mathrm{NS}$ \\
\hline
\end{tabular}




\section{Discussion}

The traditional treatment of distal radius fractures in osteoporotic patients is closed reduction and immobilization in cast. This method avoids surgery and related complications, but casts cannot maintain the length or the rotation of the distal fragment especially with comminution. (9). Loss of reduction usually occurs after two weeks of initial closed reduction ${ }^{(10)}$.

A persistent dorsal tilt results in incongruity in the distal radioulnar joint (DRUJ), and changes in the transfer of force with dorsal overload and secondary carpal bone disease ${ }^{(11)}$ Non-operative treatment - reduction and cast were producing too many unsatisfactory results, up to $30 \%$ in large surveys ${ }^{(12) .}$

In study which reported a $51.4 \%$ unsatisfactory result with closed reduction and cast in the treatment of comminuted intraarticular fracture of distal radius ${ }^{(13) .}$

Other authors have suggested that the final functional outcome in old age patients with unstable DRFs is independent of the radiographic outcome, and conservative methods had a satisfactory outcome ${ }^{(14) .}$

Considering these stated benefits of percutaneous K-wire fixation as valuable for treatment of distal end of radius fractures, the present study was planned as a comparative assessment between closed reduction casting and percutaneous K-wire fixation with restoration of radius height, inclination and volar tilt of the distal articular surface as the morphological and wrist movement as the functional outcomes.

In this systematic review aimed to compare outcomes of surgical and nonsurgical management of DRFs in persons 65 years of age or older, and the results indicate that both types of management lead to similar results with respect to DASH and VAS pain scores, as well as grip strength and most other functional assessments. While there were significant differences in wrist flexion, radial deviation, radial inclination, and ulnar variance, they did not seem to have impacts on DASH and VAS pain scores, and hence quality of life. While these results should not be interpreted that surgery for DRFs in the elderly is not necessary, considering the increasing life expectancy and low functional demands in the elderly population, maintaining good quality of life with conservative non- operative treatment may be just as important as achieving optimal recovery of hand function via surgery.

Many studies and systematic reviews performed in the past to determine the best management of DRFs in the elderly failed to reach a consensus partly due to variety of treatment options. 
One Cochrane review ${ }^{[15]}$ comparing external fixation and conservative treatment of DRFs in adults concluded that there was insufficient evidence to confirm a superior functional outcome, but external fixation did reduce displacement and provided improved anatomical results with only minor complications. Other Cochrane reviews examining surgical interventions ${ }^{[16]}$ and conservative interventions ${ }^{[17]}$ for DRFs concluded that there was insufficient evidence to determine when to perform surgery, or what type of surgical or nonsurgical management is best. A review specifically examining percutaneous pinning for DRFs found that the precise role and methods of percutaneous pinning have not been established, and that Kapandji pinning and biodegradable materials are often associated with a higher rate of complications [18].

A meta-analysis in $2011^{(19)}$ examined internal versus external fixation for unstable DRFs and found that internal fixation was associated with better grip strength, supination, and pronation, superior DASH score, and fewer total surgical complications.

A subsequent meta-analysis in $2012^{\text {[20] }}$ compared external fixation and internal fixation and reported that ORIF resulted in better functional outcomes, forearm supination, and anatomic volar tilt, whereas external fixation resulted in better grip strength and wrist flexion. A 2013 metaanalysis also found that internal fixation resulted in better functional outcomes, supination, restoration of anatomic volar tilt and radial inclination, and fewer surgical complications than external fixation.

In study done in $2009^{[21]}$ randomized patients with intra-articular DFRs to receive either external fixation or ORIF and found no differences in clinical or radio- logical outcomes or complications between the two methods In a review specifically designed to examine outcomes of unstable DRFs in patients more than 60 years of age.

In the present review there was no statistical significance difference between CI and PKF groups in grip strength in all the selected studies. Also there were no statistical significance differences between CI and PKF groups in side of lesion and classification of severity in any of the selected studies.

In study done which ${ }^{[11]}$ compared closed reduction and plaster casting, with percutaneous Kirschner-wire fixation of DFRs, and found that wiring was associated with better grip strength and hand function at 6 months with reduced risk of displacement. In study done in $2010{ }^{[23]}$ reported similar functional and radiological outcomes of 
DRFs treated operatively with percutaneous pinning and non- operative management.

In this analysis, DASH scores were reported in three of the included studies, and while the overall comparison of the surgical and cast groups showed little difference, there were slight differences between specific studies. In a randomized multi-center study

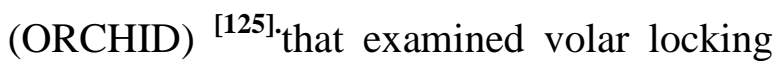
plates, reported scores of SF, EQ-5D, SF36, and DASH were not different between two treatment groups. In study which ${ }^{[15]}$ also examined volar locking plates and flexionextension at 12 months were not different between the groups, pronation-supination at 12 months showed that the ORIF group had slightly better forearm rotation, and there was no difference in DASH scores at 12 months between the groups.

In study done in $2012^{[21]}$ which studied external fixation and found no difference in DASH scores, wrist flexion, radial deviation, pronation, supination, grip strength, pinch strength (clinically), ulnar variance, and radial inclination (radiographically).

The early functional recovery observed in this cohort appears to be reflected in the fact that dynamic movement at the wrist is commenced at two weeks post-operatively. This movement was initially commenced selectively in the volar direction in a dorsally unstable fracture and gradually incorporated free activity over six weeks at the selectively lockable hinge, which was positioned at the mid-carpal level on the ulnar side of the wrist. The position of the hinge on the ulnar side avoided interference with the first carpometacarpal (CMC) joint movement and movement of the thumb. The hinge being on the ulnar side and mounted on a non-invasive device avoids the need for shifting the axis of rotation dorsally, which may have compromised results in other systems in the past since the bridging external fixator often uses invasive pins mounted on a metacarpal and the distal radius and cannot achieve placement of a hinge parallel to the axis of rotation of the joint. The early dynamisation has the potential to cause micro-movement at the fracture site, which may have contributed to rapid fracture healing at a mean of 4.4 weeks [22].

In this review there were statistical significance difference between CI and PKF groups in dorsal angulation and redial length in all of the selected studies who reported there parameters.

There was no statistical significance difference between $\mathrm{CI}$ and PKF groups frequency of complication in all of the selected studies., with BrEF resulting in the highest proportion of minor and major complications not requiring surgery, Non- 
invasive CI resulted in the lowest proportion of complications in all categories

The rate of recovery and limitations of ADL during treatment affect the quality of life of patients with DRFs. Compared to younger patients, the elderly already experience a delay of approximate 6-month in gaining functional improvement. These findings imply that rate of recovery of ADL performance and the possibility of major complications during recovery may be more important factors than the final functional outcome when deciding which treatment strategy is best for elderly patients with DRFs. A decision analysis, which compares the utility of, or preference for, each treatment option from the perspective of elderly individuals themselves, may serve as a reference for decision-making based on risk-benefit ratio that the elderly population places on each intervention ${ }^{(\mathbf{2 3})}$

Though the American Academy of Orthopedic Surgeons recommends the use of vitamin $C$ to prevent CRPS after a DRF ${ }^{\text {[24], a }}$ meta-analysis showed that it reduced the incidence of CRPS ${ }^{[187]}$ while another study has shown no benefit [138]. Other complications such as loss of reduction, surgical site infection, or mal union of fractures were more likely to be attributed to surgery. As the functional needs of the elderly are not as rigorous as in younger patients, these complications and adverse effects might exert a larger impact on the reported functional outcomes and potentially could bias against surgery.

There are a number of limitations of this study that should be considered. The number of studies meeting the inclusion criteria was small, and fewer than five studies were available for meta-analysis in most of the outcome categories, and in some categories, only two data sets were available for inclusion in the analysis. There was marked variation in the types of surgical procedures performed, and the inclusion of $\mathrm{K}$-wire fixation, external fixation, and ORIF with locking plates make the operative group heterogeneous. No analysis of different types of distal radius fractures or different types of surgical intervention was performed, nor did we examine the effects of complications caused by these two broadly defined types of intervention. Follow-up time of the studies also varied, as did patient selection criteria. Furthermore, this study only focused on the elderly population, so the findings may not apply to younger population whose functional demands of their hands are higher and might have reported different out- come scores.

In summary, the results of this systematic review suggest that nonsurgical management can be a valid treatment option for DRFs in 
elderly patients. However, they should not be interpreted as a replacement for surgery, as operations should still be performed where there are surgical indications. But when there are no definitive surgical indications, nonsurgical management can avoid postoperative complications and brings good quality of life. While certain objective functional outcomes may be better with surgical management, it is possible that they were less important to the elderly whose functional needs are not as great as in younger patients.

\section{Conclusion}

Surgical treatment seems to be more effective distal radius fracture compared with conservative treatment when the radiographic outcomes were analyzed, and no significant differences were detected in the functional outcomes and complication rate.

\section{References}

[1] V. Gauresh, Distal end radius fractures: evaluation of results of various treatments and assessment of treatment choice. Chin J Traumatol, vol. 17, no. 4, pp. 214-219, 2014.

[2] J. Song, Yu AX, Li ZH, Comparison of conservative and operative treatment for distal radius fracture: a meta-analysis of randomized controlled trials. Int J Clin Exp Med, vol. 8, no. 10, pp. 17023-17035, 2015.
[3] EK. Shin and JB. Jupiter, Current Concepts in the Management of Distal Radius Fractures. Acta Chirg Orthop Traumatol, vol. 74, pp. 233-246, 2007.

[4] K. Ipaktchi, M. Livermore, C. Lyons, R. Banegas, Current Concepts in the Treatment of Distal Radial Fractures. Trauma Update, vol. 36, no. 10, pp. 778-785, 2013.

[5] WG. Blakeney, Stabilization and treatment of Colles' fractures in elderly patients. Clin Intervent in Aging, vol. 5, pp. 337-344, 2010.

[6] PC. Rhee, DG. Dennison, S. Kakar, Avoiding and treating perioperative complications of distal radius fractures. Hand Clin, vol. 28, no. 2, pp. 185-98, 2012.

[7] DRJ. Garcia and KC. Chung, Common myths and evidence in the management of distal Radius fractures. Hand Clin, vol. 28, no. 2, pp. 127-133, 2012.

[8] M. Soong, BE. Earp, G. Bishop, A. Leung, P. Blazar, Volar Locking Plate implant prominence and flexor tendon rupture. J Bone Joint Surg Am, vol. 93, no. 4, pp. 328-335, 2011.

[9] T.J. Walton, J.Brammar, D.Hutchinson, Treatment of unstable distal radial fractures by intrafocal, intramedullary K-wires Coleman Injury, Int. J. Care Injured, pp. 383-389, 2001.

[10] Weil WM, Trumble TE, Treatment of distal radius fractures with intrafocal (kapandji) pinning and supplemental skeletal stabilization: Hand Clin Aug, vol. 21, no. 3, pp. 317-28, 2005.

[11] Lolade Giwa, Kate Spacey, Greg Packer. Management of a Complex, Multioperated IntraArticular Distal Radius Fracture, J Wrist Surg Aug, vol. 4, no. 3, pp. 179-182, 2015.

[12] Thomas BP, Sreekanth R. Distal radioulnar joint injuries. Indian J Orthop Sep, vol. 46, no. 5, pp. 493-504, 2012. 
[13] A. Daniel. Rikli, Adrian Businger, Reto Babst, Dorsal double-plate fixation of the distal radius. Operat Orthop Traumatol, vol. 17, pp. 624-40, 2005

[14] E. Spira, K. Weigl, The comminuted fracture of the distal end of the radius. Reconstr Surg Traumatol, vol. 11, pp. 128-38, 1968.

[15] BT. Young, GM. Rayan, Outcome following non operative treatment of displaced distal radius fractures in low-demand patients older than 60 years. J Hand Surg [Am], vol. 25, pp. 19, 2000.

[16] HH. Handoll, JS. Huntley, R. Madhok, External fixation versus conservative treatment for distal radial fractures in adults. Cochrane Database Syst Rev, vol. 3, pp. CD006194, 2007.

[17] HH. Handoll, R. Madhok, Surgical interventions for treating distal radial fractures in adults. Cochrane Database Syst Rev, vol. 3, pp. CD003209, 2003.

[18] HH. Handoll, R. Madhok, Conservative interventions for treating distal radial fractures in adults. Cochrane Database Syst Rev, vol. 2, pp. CD000314, 2003.

[19] Z. Cui, J. Pan, Internal versus external fixation for unstable distal radius fractures: an up-to-date meta-analysis. Int Orthop, vol. 35, pp. 1333-1341, 2011.

[20] DH. Wei, RW. Poolman, M. Bhandari, VM. Wolfe, MP. Rosenwasser External fixation versus internal fixation for unstable distal radius fractures: a systematic review and meta-analysis of compar- ative clinical trials. J Orthop Trauma, vol. 26, pp. 386-394, 2012.

[21] R. Arora, M. Gabl, M. Gschwentner, C. Deml, A comparative study of clinical and radiologic outcomes of unstable colles type distal radius fractures in patients older than 70 years: nonoperative treatment versus volar locking plating. J Orthop Trauma, vol. 23, pp. 237-242, 2009.

[22] Francesco Franceschi, Edoardo Franceschetti Michele, Volar locking plates versus K-wire/pin fixation for the treatment of distal radial fractures: a systematic review and quantitative synthesis British Medical Bulletin, September, vol. 115, pp. 91-110, 2015.

[23] M. Grabicki, A. Grzegorzewski, Evaluation of distal radius fracture treatment-non-operative vs. operative with percutaneous pinning. Chir Narzadow Ruchu Ortop Pol, vol. 75, pp. 205-210 [Article in Polish], 2010.

[24] C. Bartl, D. Stengel, T. Bruckner, The treatment of displaced intra-articular distal radius frac- tures in elderly patients. Dtsch Arztebl Int, vol. 111, pp. 779-787, 2014.

To cite this article: Mohammed Al-Morsi, Mohamed Ebrahim, Abdelfatah El-Senosy Conservative Treatment versus Percutaneus Wire Fixation in Treatment of Distal Radial Fracture in Elderly. BMFJ 2020; 37 (surgical issue):20-32. DOI: 10.21608/bmfj.2020.21966.1201 